\title{
An Energy-Dependent Numerical Model for the Condensation Probability, $\gamma_{j}$
}

\author{
Leslie M. Kerby ${ }^{\mathrm{a}, \mathrm{b}}$ \\ ${ }^{a}$ Idaho State University, Pocatello, ID 83209, USA \\ ${ }^{b}$ Los Alamos National Laboratory, Los Alamos, NM 87545, USA
}

\begin{abstract}
The "condensation" probability, $\gamma_{j}$, is an important variable in the preequilibrium stage of nuclear spallation reactions. It represents the probability that $p_{j}$ excited nucleons (excitons) will "condense" to form complex particle type $j$ in the excited residual nucleus. It has a significant impact on the emission width, or probability of emitting fragment type $j$ from the residual nucleus. Previous formulations for $\gamma_{j}$ were energy-independent and valid for fragments up to ${ }^{4} \mathrm{He}$ only. This paper explores the formulation of a new model for $\gamma_{j}$, one which is energy-dependent and valid for up to ${ }^{28} \mathrm{Mg}$, and which provides improved fits compared to experimental fragment spectra.
\end{abstract}

Keywords: condensation probability, nuclear spallation reactions, Monte Carlo, transport codes, MCNP6, cascade exciton model (CEM), fragment spectra

\section{Introduction}

The Computational Physics group 3 (Monte Carlo Codes) within the $\mathrm{X}$ Division at Los Alamos National Laboratory (LANL) has led the development of the transport code MCNP6 (Monte Carlo N-Particle transport code, version 6) [1]. MCNP6 is a general-purpose, continuous-energy, generalized-geometry, time-dependent, Monte Carlo radiation-transport code designed to track many particle types over broad ranges of energies. It is used around the world in applications ranging from radiation protection and dosimetry, nuclear reactor design, nuclear criticality safety, detector design and analysis, decontamination and decommissioning, accelerator applications, medical physics, to space research and beyond. At higher energies ( $>150 \mathrm{MeV}$ ) MCNP6 uses the Cascade Exciton Model version 03.03 (CEM03.03) [2, 3] and the Los Alamos Quark-Gluon String Model version 03.03 (LAQGSM03.03) [3, 4] to model nuclear reactions.

CEM and LAQGSM model a nuclear spallation reaction as generally passing through three stages: the intranuclear cascade and accompanying coalescence, the preequilibrium, and lastly evaporation/fission. The "condensation" probability, $\gamma_{j}$, is an important variable in the preequilibrium stage. It represents the probability that $p_{j}$ excited nucleons (excitons) will "condense" to form complex particle type $j$ in the excited residual nucleus. It has a significant impact on the emission width, or probability of emitting fragment type $j$ from the residual nucleus [5-7]. This paper explores the formulation of a new model for $\gamma_{j}$, one which is energy-dependent, and which provides improved fits compared to experimental fragment spectra.

This $\gamma_{j}$ model is specifically designed for use in CEM and MCNP6, taking into account the reaction mechanisms used (or

Email address: kerblesl@isu.edu (Leslie M. Kerby) not used) in CEM. However, this model could be useful in other nuclear spallation codes and models, especially for heavy cluster production.

\section{Background}

This $\gamma_{j}$ model is part of a larger project [8-12] aimed at producing high-energy light fragments (LF) in nuclear spallation reactions simulated with CEM03.03 and LAQGSM03.03, which are the default event generators within MCNP6 for highenergy nuclear reactions ( $>150 \mathrm{MeV})$.

The preequilibrium interaction stage of nuclear reactions is considered by the current CEM and LAQGSM in the framework of the latest version of the Modified Exciton Model (MEM) [13, 14] as described in Ref. [15]. At the preequilibrium stage of a reaction, we take into account all possible nuclear transitions changing the number of excitons $n$ with $\Delta=+2,-2$, and 0 , as well as all possible multiple subsequent emissions of $\mathrm{n}, \mathrm{p}, \mathrm{d}, \mathrm{t},{ }^{3} \mathrm{He}$, and ${ }^{4} \mathrm{He}$, for the current version CEM03.03. The latest upgrades, called version CEM03.03F, include an expansion of the preequilibrium stage to allow for emission of heavy clusters up to ${ }^{28} \mathrm{Mg}$. The corresponding system of master equations describing the behavior of a nucleus at the preequilibrium stage is solved by the Monte-Carlo technique [2]. We had previously modeled $\gamma_{j}$ for emitted fragments up to ${ }^{4} \mathrm{He}$; with the expansion of the preequilibrium to include production of heavy clusters up to ${ }^{28} \mathrm{Mg}$, a new model for $\gamma_{j}$ that included these heavy clusters was required.

The new CEM03.03F considers the possibility of fast heavy cluster emission at the preequilibrium stage of a reaction in addition to the emission of nucleons and light fragments up to ${ }^{4} \mathrm{He}$. We assume that in the course of a reaction $p_{j}$ excited nucleons (excitons) are able to condense with probability $\gamma_{j}$ forming a complex particle which can be emitted during the preequi- 
librium state. This "condensation" probability $\gamma_{j}$ was originally proposed by Ribansky and Oblozinsky [16] as an improvement over an empirical factor introduced by Cline in his emissionwidth formulation [6]. Ribansky theorized that $\gamma_{j}$ could be calculated from first principles, considering the specific configuration of distribution of the energy $E$ among $n$ excitons in the residual nucleus and the specific particle $j$ to be formed, but such a calculation is not feasible given current Monte Carlo computation time limitations. $\gamma_{j}$ is, therefore, estimated as the overlap integral of the wave function of independent nucleons with that of the complex particle (see details in [2]):

$$
\gamma_{j} \simeq p_{j}^{3}\left(p_{j} / A\right)^{p_{j}-1} .
$$

This is a rather crude estimate. As is frequently done (see e.g., Refs. $[17,18])$, the values of $\gamma_{j}$ are taken from fitting the theoretical preequilibrium spectra to the experimental ones. In CEM, to improve the description of preequilibrium complexparticle emission, we estimate $\gamma_{j}$ by multiplying the estimate provided by Eq. 1 by an empirical coefficient $F_{j}\left(A, Z, T_{0}\right)$ whose values are fitted to available nucleon-induced experimental complex-particle spectra. Therefore, the new equation for $\gamma_{j}$ with this coefficient is shown in Eq. 2:

$$
\gamma_{j}=F_{j} p_{j}^{3}\left(\frac{p_{j}}{A}\right)^{p_{j}-1} .
$$

Values of $F_{j}$ for d, t, ${ }^{3} \mathrm{He}$, and ${ }^{4} \mathrm{He}$ needed to be re-fit after the upgrades to the inverse cross section and coalescence models; and new values of $F_{j}$ needed to be obtained for heavy clusters, up to ${ }^{28} \mathrm{Mg}$, after we expanded preequilibrium emission to include these heavy clusters.

Previously, $\gamma_{j}$ had been formulated with no energy dependence $[5,6,16]$. We accordingly expect energy to be the largest factor in the model for $F_{j}$. Lastly, the importance of $\gamma_{j}$ can be seen in the calculation of the emission width, $\Gamma_{j}$, for complex fragments, represented by Eq. 3:

$$
\begin{aligned}
\Gamma_{j}(p, h, E)= & \int_{V_{j}^{c}}^{E-B_{j}} \gamma_{j} \frac{2 s_{j}+1}{\pi^{2} \hbar^{3}} \mu_{j} \mathfrak{R}(p, h) \frac{\omega\left(p_{j}, 0, T+B_{j}\right)}{g_{j}} \\
& \times \frac{\omega\left(p-p_{j}, h, E-B_{j}-T\right)}{\omega(p, h, E)} T \sigma_{j}^{i n v}(T) d T,
\end{aligned}
$$

where:

$p$ is number of particle excitons;

$h$ is number of hole excitons;

$E$ is internal energy of the excited nucleus (sometimes referred to as $U$ );

$B_{j}$ is the binding energy of particle $\mathrm{j}$;

$V_{j}^{c}$ is Coulomb barrier of particle $\mathrm{j}$;

$\gamma_{j}$ is probability that the proper number of particle excitons will coalesce to form a type $j$ fragment (also called $\gamma_{\beta}$ in a number of early publications; see, e.g., Refs. [5, 17, 18]); $s_{j}$ is the spin of the emitted particle $j$;

$\mu_{j}$ is the reduced mass of the emitted particle $j$;

$\mathfrak{R}$ creates zero probability of emission if the number of particle excitons is less than the number of nucleons in particle $j$; $\omega$ is the level density of the $n$-exciton state;

$T$ is the kinetic energy of the emitted particle $j$;

$\sigma_{i n v}$ is the inverse cross section.

The values for $\gamma_{j}$ directly impact the emission width, which in turn determines the amount of fragment production.

\section{Statistical Analysis}

An increase or decrease in $F_{j}$ generally leads to an increase or decrease in the emission of fragment type $j$, especially in the high-energy tails. We fit these values of $F_{j}$ so that the CEM03.03F results matched experimental data as closely as possible. The $F_{j}$ values we obtained, for several hundred reactions, are available in Appendix A of Ref. [19]. The results for predicted fragment spectra using these $F_{j}$ fitted values were plotted against experimental data and predicted results from the original CEM03.03, for the several hundred reactions we fit, and are available in [20]. We do not show them here to conserve space.

With the data set complete, we can analyze it with the statistical programming language $R[21]$. We first look at $F_{j}$ values for proton-induced reactions. Fig. 1 displays values of $F_{j}$ according to incident proton energy. Two effects become apparent in this graph. First, $F_{j}$ appears to have an exponentially

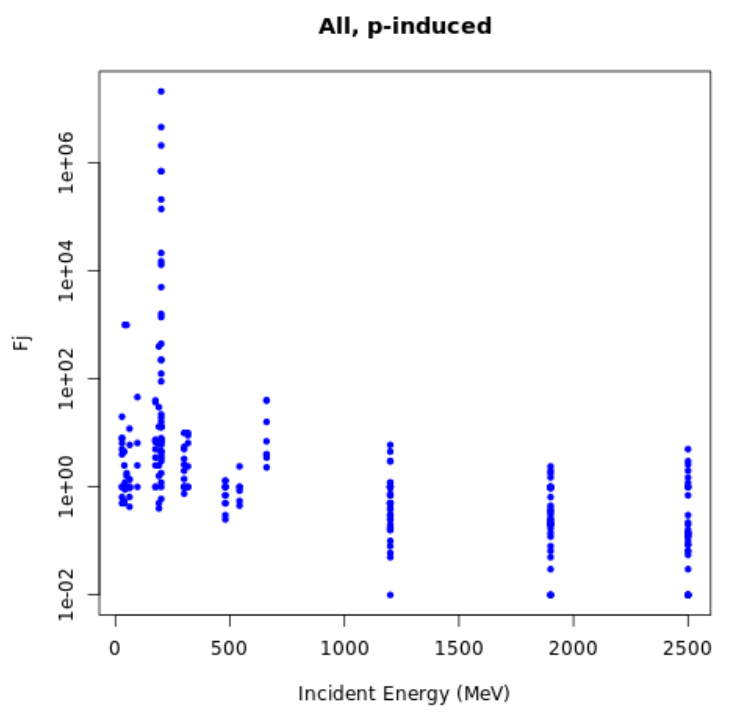

Figure 1: Values of $F_{j}$ according to incident proton energy (MeV).

decreasing energy dependence. This energy dependence makes sense considering the physics of nuclear reactions. We consider in this $F_{j}$ model the incident energy of the incoming proton, not the excitation energy of the residual nucleus at the time of preequilibrium, and after a certain incident energy we expect the residual nucleus energy to not change significantly as the incident energy continues to increase: i.e., the amount of energy deposited in the target nucleus reaches a saturating limit and increasing the incident proton energy further does not lead to significantly greater residual nucleus energy. This idea is 
analogous to "limiting fragmentation" [22, 23]. In addition, CEM accounts for the IntraNuclear Cascade, preequilibrium, evaporation/fission, Fermi break-up, and coalescence mechanisms of nuclear reactions, but does not account for pick-up and knock-out reactions. Pick-up and knock-out mechanisms are especially important at low energies, and therefore, the increase in $F_{j}$ at lower incident energies can be attributed to "compensating" for these missing physics in CEM. Furthermore, CEM does not account for some nuclear structure effects important at low energies, and for some reactions, also at high energies. Lastly, CEM, just as any other model, is "only a model," and probably misses some other aspects of the physics. For these reasons, we need to look at the "model" for $\gamma_{j}$ in CEM as heuristic, understanding that in some energy/target-size regions, it does not have exactly the right meaning of the condensation probability, but also contains a component to counterbalance physics not accounted for in CEM.

The second effect we see from Fig. 1 are the "stacks" of $F_{j}$ values at each energy; we will see that these correspond to different emitted fragment sizes, and therefore $F_{j}$ is also dependent on the emitted fragment. Fig. 2 displays the incidentenergy dependence of $F_{j}$ for select emitted fragments individually. The "stacks" which occur in Fig. 2 are largely attributable to varying target sizes.

Fig. 3 displays the $F_{j}$ values according to target atomic size. From this graph there is no clear dependence on target mass number. However, we suspected that if we separated out the energy dependence and fragment-size dependence, that we could uncover a dependence on target mass number. We first plotted $F_{j}$ values by both energy and target mass number (see Fig. 4). We zoomed in on $F_{j}$ values $<1000$, as there were some very large values for $F_{j}$ which made it difficult to see any pattern. The color darkens with increasing $A_{t}$ (target mass number), and is intended to aid the reader in discerning depth. In Fig. 4 we can begin to see a target-size dependence, but it is still somewhat obscured by including all different emitted fragment sizes. We also plotted $F_{j}$ by target size for each emitted fragment type (see Fig. 5 for a few examples). Interestingly, in these plots $F_{j}$ appears to decrease as target size increases, but we must be careful because these results are largely obscured by including all different incident energies. We will see that incident energy is the dominant variable in $F_{j}$, and therefore by including all incident energies we cannot draw any conclusions about variables of secondary importance. When we plot $F_{j}$ by both incident energy and target size for each individual emitted fragment, we see a different pattern emerge: namely that $F_{j}$ increases as target size increases (see Figs. 8-10).

We therefore have established that $F_{j}$ is dependent upon incident energy, target size, and fragment size. We assume for mathematical simplicity that the dependencies on incident energy and target size are separable, and that the target-size dependence is not dependent upon fragment size. We ignore any separate effect of $Z_{t}$, the charge number of the target, as it is highly correlated with the target mass number $A_{t}$ and we assume the inclusion of $A_{t}$ is sufficient to capture the overall effect. However, the separate dependencies on $A_{t}$ and $Z_{t}$ have not been studied thoroughly, and could be explored in future work.
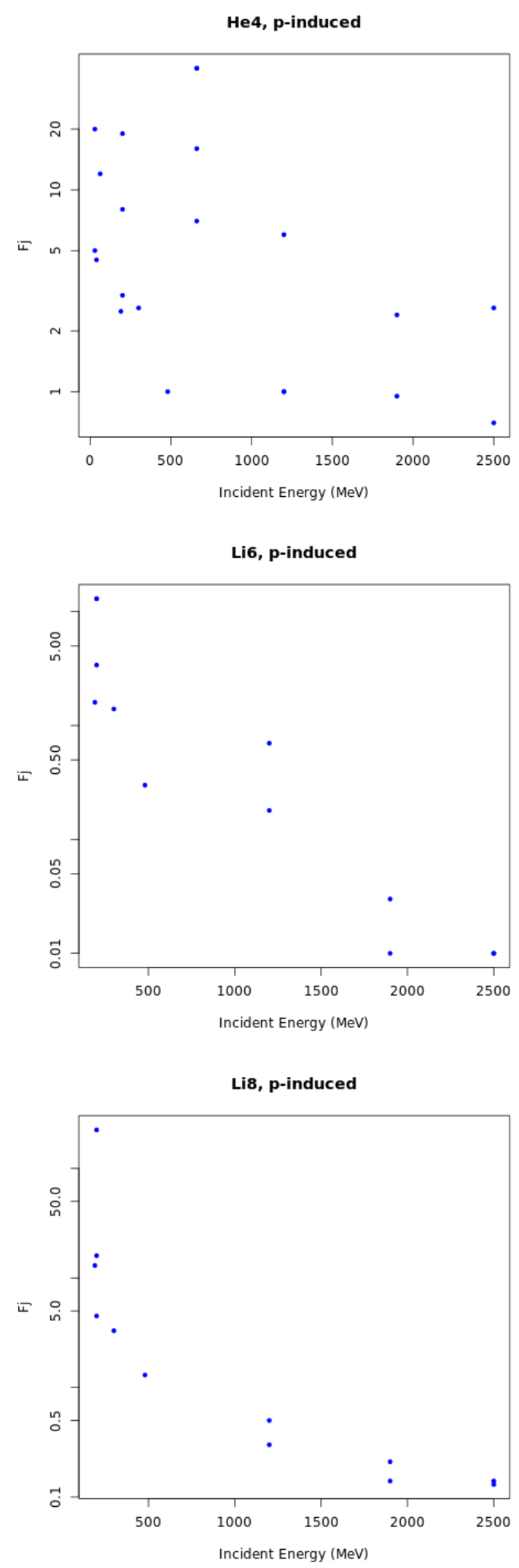

Figure 2: Values of $F_{j}$ according to incident proton energy (MeV), for emitted fragments ${ }^{4} \mathrm{He},{ }^{6} \mathrm{Li}$, and ${ }^{8} \mathrm{Li}$. 


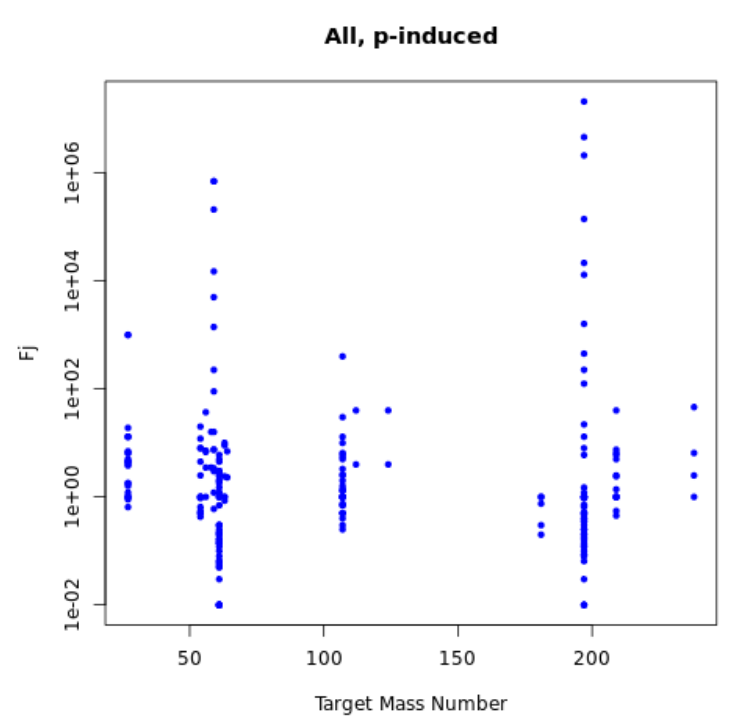

Figure 3: Values of $F_{j}$ according to the mass number of the target.

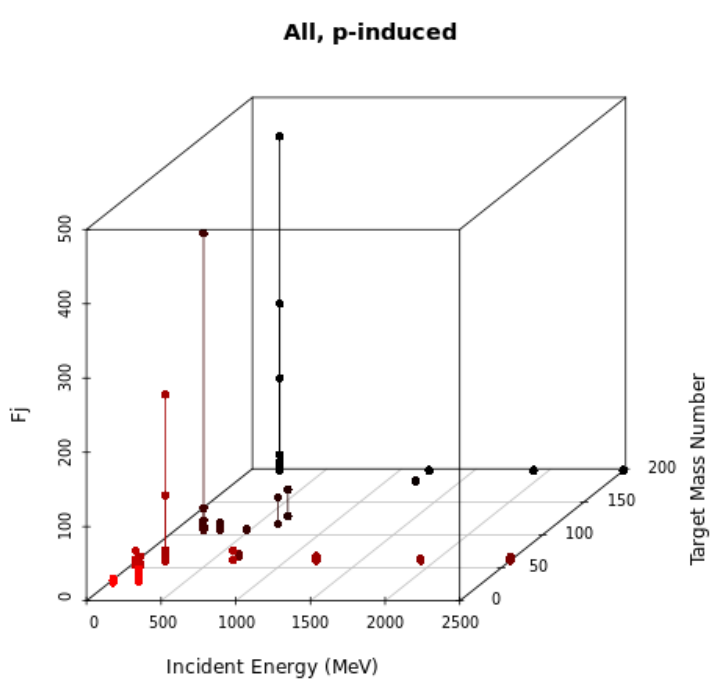

Figure 4: Values of $F_{j}$ according to the incident proton energy (MeV) and mass number of the target.

\subsection{Fragment-Specific $F_{j}$}

As discussed in the previous section, we assume an $F_{j}$ model can be obtained which has the form of Eq 4.

$$
F_{j}\left(T_{0}, A_{j}, Z_{j}, A_{t}\right)=f\left(T_{0}, A_{j}, Z_{j}\right) g\left(A_{t}\right),
$$

$T_{0}$ : Incident energy of projectile (MeV);

$A_{j}$ : Mass number of emitted fragment type $j$;

$Z_{j}$ : Atomic number of emitted fragment type $j$;

$A_{t}$ : Mass number of target nucleus.

A simple exponential decay described $g\left(A_{t}\right)$ quite well. We developed it such that it is valid for all $A_{t}<300$. A suitable model for $f\left(T_{0}, A_{j}, Z_{j}\right)$ proved more difficult to obtain. All common

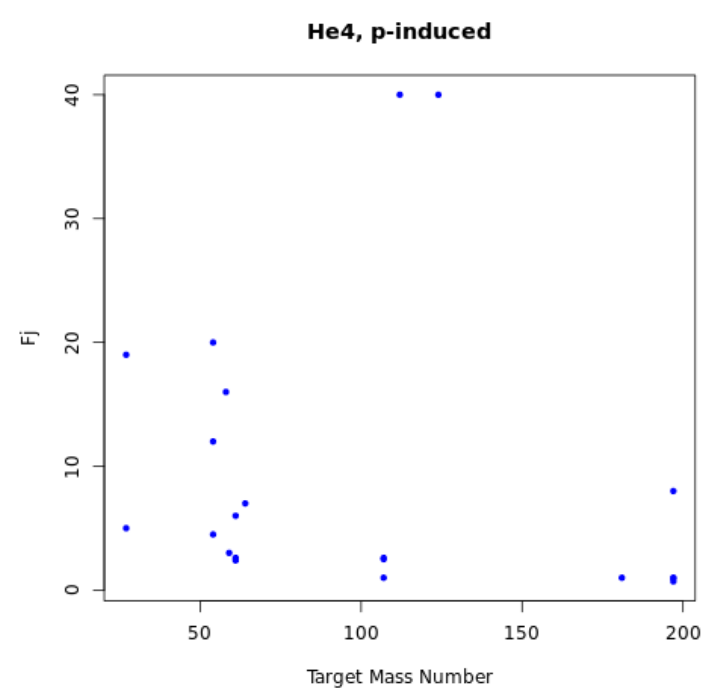

Li6, p-induced

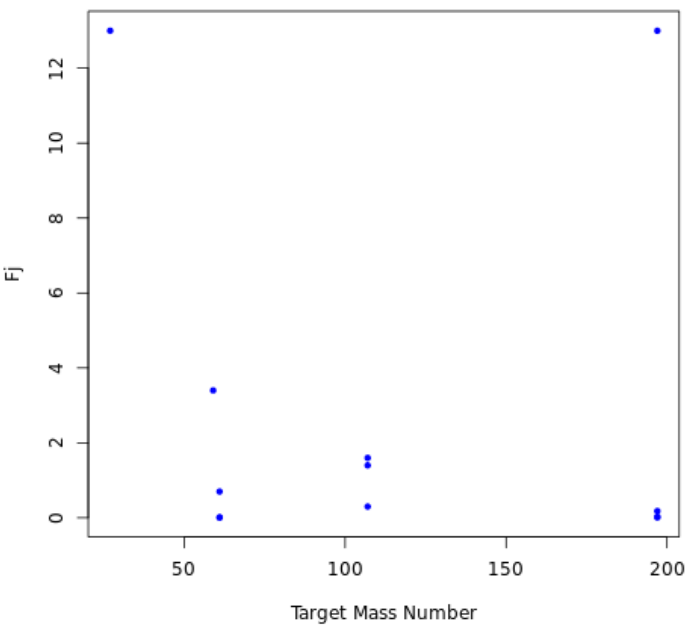

Li6, p-induced

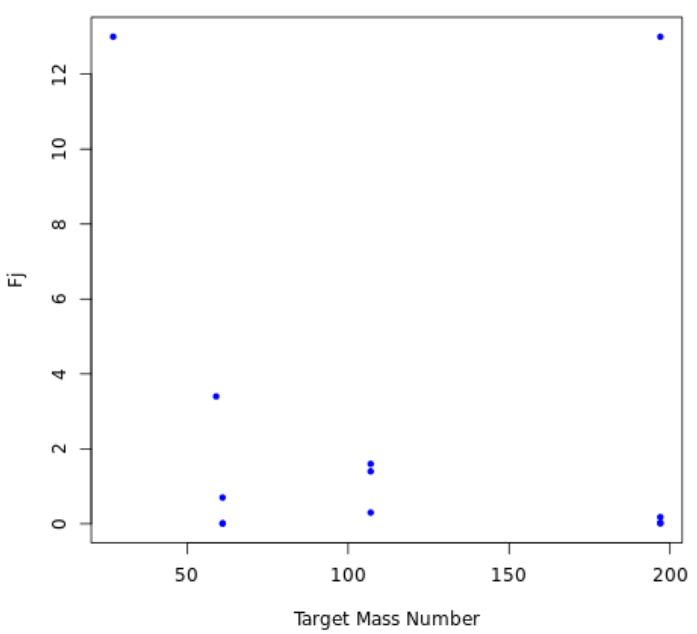

Figure 5: Values of $F_{j}$ according to target mass number, for emitted fragments ${ }^{4} \mathrm{He},{ }^{6} \mathrm{Li}$, and ${ }^{8} \mathrm{Li}$ 
distributions were tested and none of them were able to describe both the low-energy and high-energy dependencies of $F_{j}$. We therefore used one function (an exponential decay) to describe the low-energy dependence and a second function (a $1 / T_{0}$ term) to describe the high-energy dependence. For the $1 / T_{0}$ term, we added 100 to the denominator to ensure no singularities (code crashes) in the range of conceivable energies. These two different energy dependencies make sense considering the previous discussion about CEM lacking some important physics in the low-energy region, and therefore the $F_{j}$ model is "compensating" for missing physics in that region, but in the higher-energy region CEM is reliable enough. We also found that for light LF $\left({ }^{4} \mathrm{He}\right.$ and lighter $)$, dependence on target size disappeared. Equations for specific fragment types are shown in Eq. 5:

$$
\begin{aligned}
& F_{d}=-3 e^{-T_{0} / 20}+\frac{125}{T_{0}^{0.2}+100} ; \\
& F_{t}=20 e^{-T_{0} / 20}+\frac{175}{T_{0}^{0.4}+100} ; \\
& F^{3} \mathrm{He}=20 e^{-T_{0} / 20}+\frac{250}{T_{0}^{0.6}+100} ; \\
& F_{{ }^{4} \mathrm{He}}=100 e^{-T_{0} / 20}+\frac{1000}{T_{0}^{0.8}+100} ; \\
& F_{6} \mathrm{He}=\left[1.4 e 5 e^{-T_{0} / 20}+\frac{1400}{T_{0}^{1.2}+100}\right] e^{-\frac{300-A_{t}}{100}} ; \\
& F_{6} L i=\left[4.0 e 5 e^{-T_{0} / 20}+\frac{5000}{T_{0}^{1.2}+100}\right] e^{-\frac{300-A_{t}}{100}} ; \\
& F_{7} L i=\left[1.0 e 6 e^{-T_{0} / 20}+\frac{2.5 e 4}{T_{0}^{1.4}+100}\right] e^{-\frac{300-A_{t}}{100} ;} \\
& F_{8} L i=\left[2.5 e 6 e^{-T_{0} / 20}+\frac{1.0 e 5}{T_{0}^{1.6}+100}\right] e^{-\frac{300-A_{t}}{100} ;} \\
& F_{9} L i=\left[6.25 e 6 e^{-T_{0} / 20}+\frac{4.0 e 5}{T_{0}^{1.8}+100}\right] e^{-\frac{300-A_{t}}{100}} ; \\
& F_{7} B e=\left[1.0 e 6 e^{-T_{0} / 20}+\frac{5000}{T_{0}^{1.2}+100}\right] e^{-\frac{300-A_{t}}{100}} ; \\
& F_{9_{B e}}=\left[6.25 e 6 e^{-T_{0} / 20}+\frac{1.0 e 5}{T_{0}^{1.6}+100}\right] e^{-\frac{300-A_{t}}{100}} ; \\
& F_{{ }^{10}} B e=\left[1.56 e 7 e^{-T_{0} / 20}+\frac{4.0 e 5}{T_{0}^{1.8}+100}\right] e^{-\frac{300-A_{t}}{100}} ; \\
& F_{{ }^{10} B}=\left[1.56 e 7 e^{-T_{0} / 20}+\frac{1.5 e 5}{T_{0}^{1.6}+100}\right] e^{-\frac{300-A_{t}}{100}} ; \\
& F_{{ }_{11} B}=\left[3.9 e 7 e^{-T_{0} / 20}+\frac{6.0 e 5}{T_{0}^{1.8}+100}\right] e^{-\frac{300-A_{t}}{100}} ; \\
& F_{12 B}=\left[9.75 e 7 e^{-T_{0} / 20}+\frac{2.4 e 6}{T_{0}^{2.0}+100}\right] e^{-\frac{300-A_{t}}{100}} ; \\
& F^{12} C=\left[9.75 e 7 e^{-T_{0} / 20}+\frac{6.0 e 5}{T_{0}^{1.8}+100}\right] e^{-\frac{300-A_{t}}{100}} ; \\
& F_{13} C=\left[2.44 e 8 e^{-T_{0} / 20}+\frac{2.4 e 6}{T_{0}^{2.0}+100}\right] e^{-\frac{300-A_{t}}{100}} .
\end{aligned}
$$

Examples of several fits are shown in Figs. 6-10. As men- tioned previously, in the top plot in these figures the color darkens with increasing $A_{t}$ (target mass number) to aid the reader in discerning depth. The bottom plots have no such color variation so as to remain easily distinguishable from the functional model approximation. More results are available in [20].
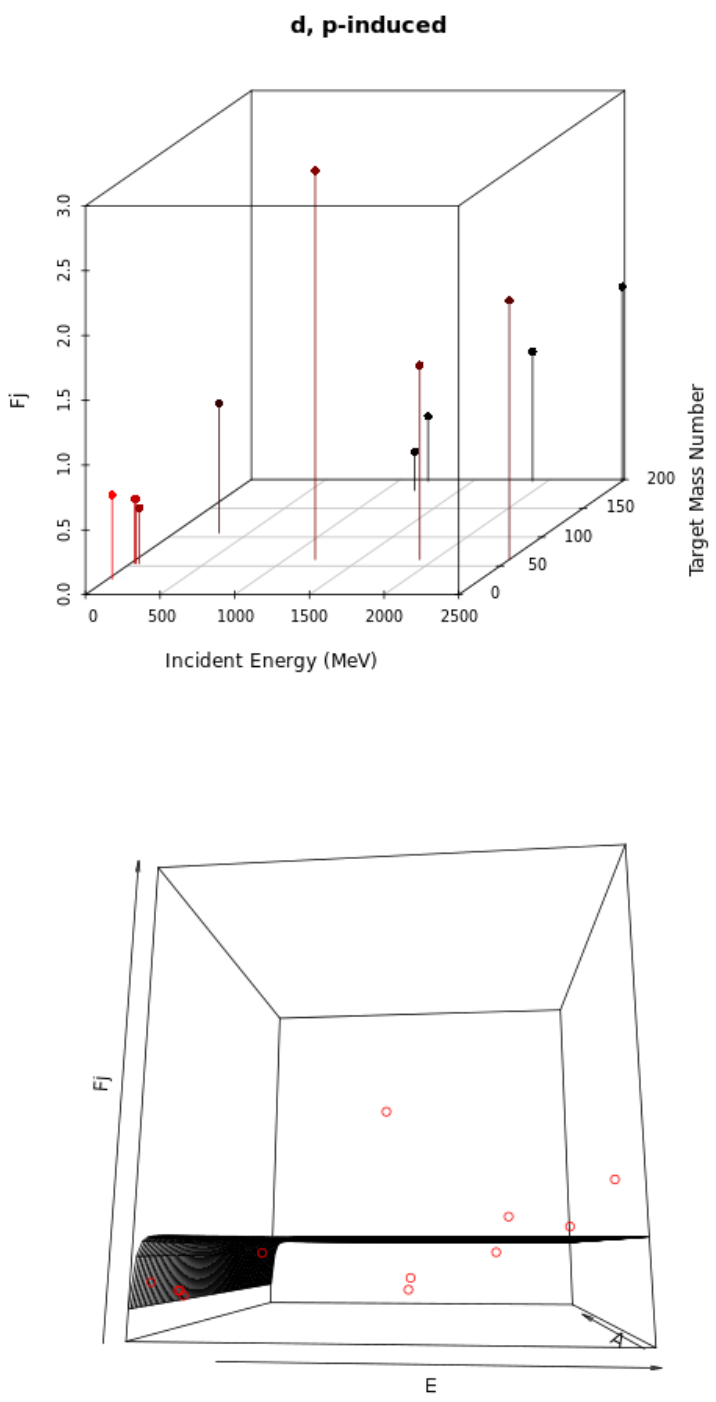

Figure 6: Values of $F_{j}$ according to incident proton energy (MeV) and target mass number (top plot), shown with a corresponding surface mesh of the $F_{j}$ model, for deuterium.

\subsection{Neutron-Induced Reactions}

Having obtained a fragment-specific $F_{j}$ model for protoninduced reactions, we turned our attention to neutron-induced reactions. There is less experimental data available for neutroninduced reactions, due to the difficulty of conducting these experiments. There is also no experimental data on the emission of light fragments heavier than ${ }^{4} \mathrm{He}$, in an energy range of interest to us in this paper. Therefore, for light fragments heavier than ${ }^{4} \mathrm{He}$ we use the fragment-specific $F_{j}$ model we obtained 

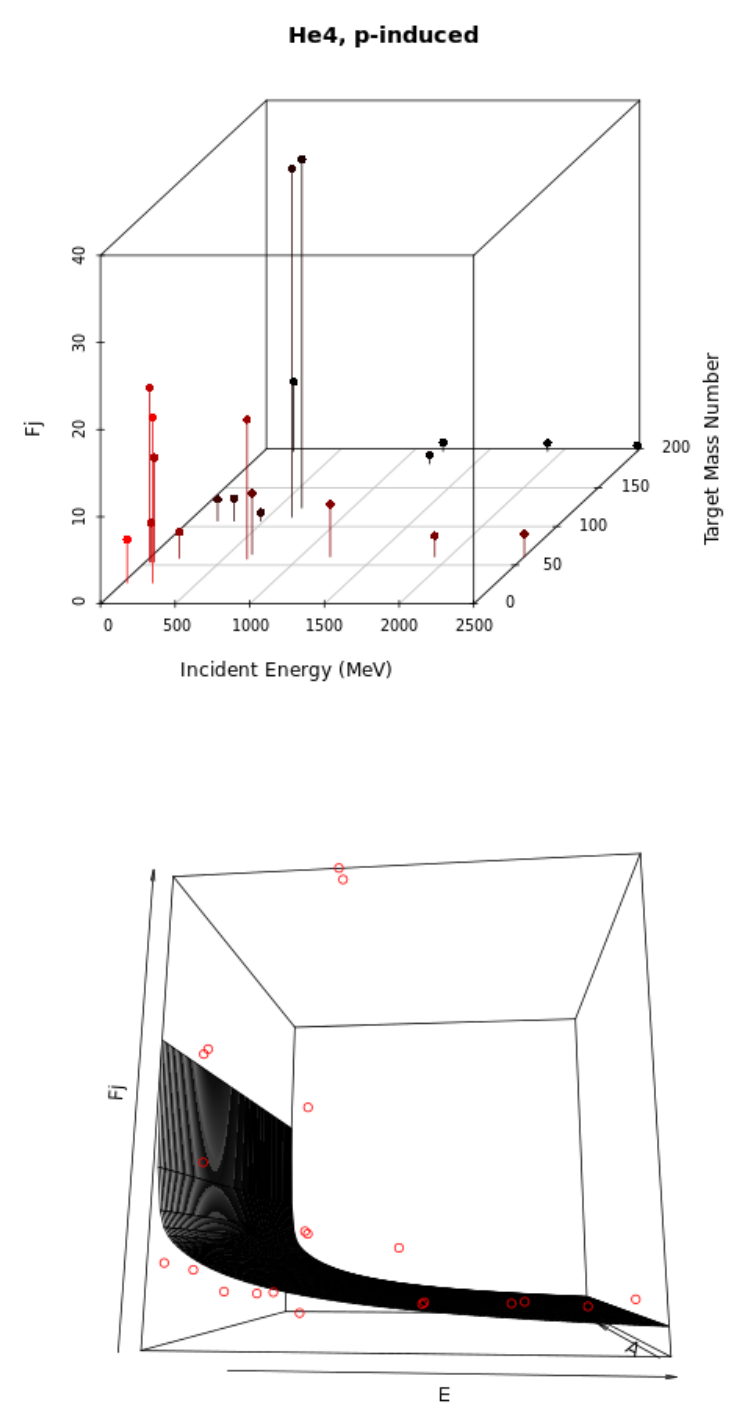

Figure 7: Values of $F_{j}$ according to incident proton energy (MeV) and target mass number (top plot), shown with a corresponding surface mesh of the $F_{j}$ model, for ${ }^{4} \mathrm{He}$.

for proton-induced reactions as a "first guess." The $F_{j}$ equations for $\mathrm{d}, \mathrm{t},{ }^{3} \mathrm{He}$, and ${ }^{4} \mathrm{He}$ for neutron-induced reactions are presented in Eq. 6, and are similar to the respective $F_{j}$ equations obtained for these emitted fragments for proton-induced reactions.

$$
\begin{gathered}
F_{d}=-4.5 e^{-T_{0} / 20}+\frac{187.5}{T_{0}^{0.2}+100} \\
F_{t}=-6.75 e^{-T_{0} / 20}+\frac{281}{T_{0}^{0.2}+100} \\
F^{{ }^{3} H e}=30 e^{-T_{0} / 20}+\frac{375}{T_{0}^{0.6}+100} \\
F_{{ }^{4} \mathrm{He}}=500 e^{-T_{0} / 20}+\frac{5000}{T_{0}^{0.8}+100}
\end{gathered}
$$

Examples of two fits are shown in Figs. 11 and 12.
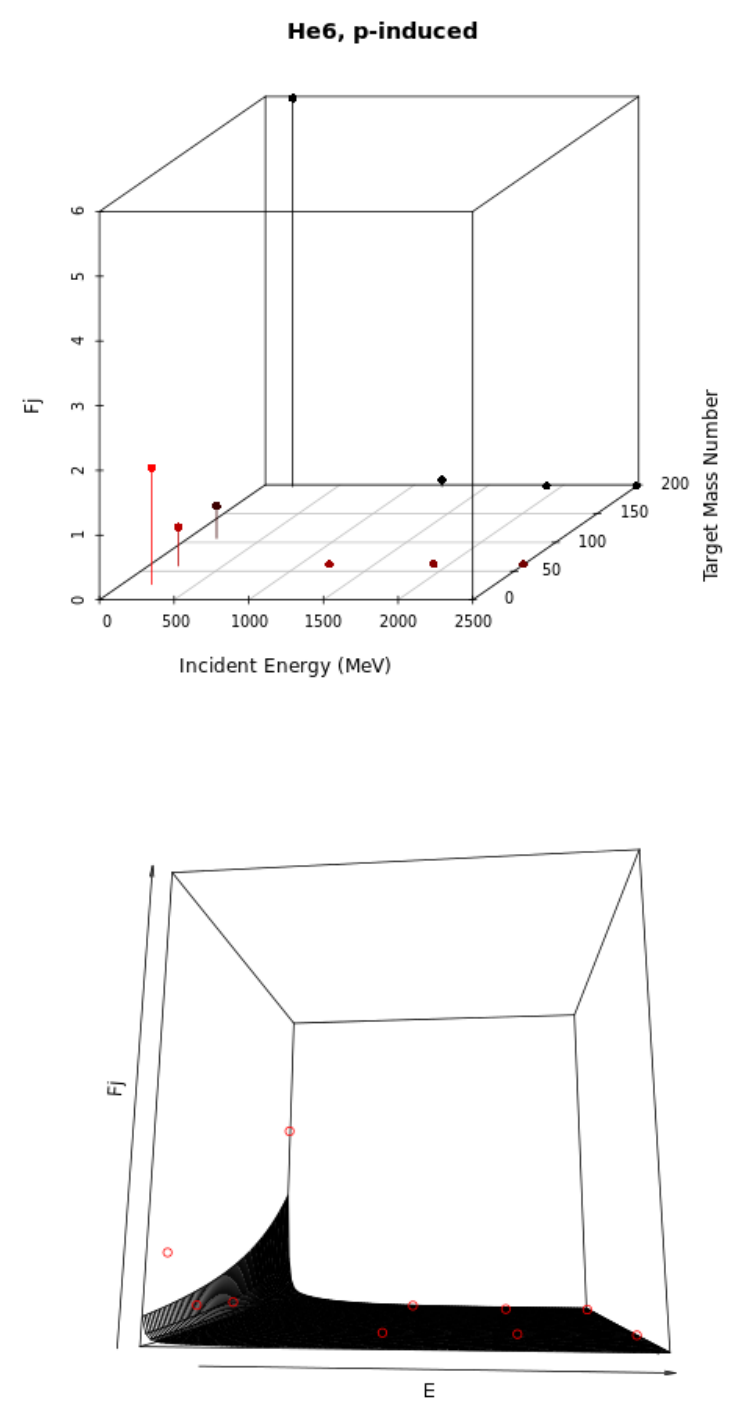

Figure 8: Values of $F_{j}$ according to incident proton energy (MeV) and target mass number (top plot), shown with a corresponding surface mesh of the $F_{j}$ model, for ${ }^{6} \mathrm{He}$.

\subsection{General $F_{j}$ model}

In studying the fragment-specific equations for $F_{j}$ in Eq. 5, a pattern quickly emerges. Heavy clusters can be nicely generalized as approximately Eq. 7:

$$
\begin{array}{r}
F_{j}\left(T_{0}, A_{j}, Z_{j}, A_{t}\right)=\left[7800(2.5)^{A_{j}} e^{-T_{0} / 20}+\frac{2(4)^{\tau}}{T_{0}^{0.2 \tau}+100}\right] \\
\times e^{-\frac{300-A_{t}}{100}}, \\
\tau=A_{j}-\left(Z_{j}-3\right) .
\end{array}
$$

This general form is used for heavy clusters for which we do not have sufficient data: ${ }^{8} \mathrm{He},{ }^{11,12} \mathrm{Be},{ }^{8,13} \mathrm{~B},{ }^{10,11,14,15,16} \mathrm{C}$, and all fragments with $Z \geq 7$ (up to ${ }^{28} \mathrm{Mg}$ ).

Recall that $\gamma_{j}$ can theoretically be calculated from first principles, but that this is too computationally time-consuming. We 

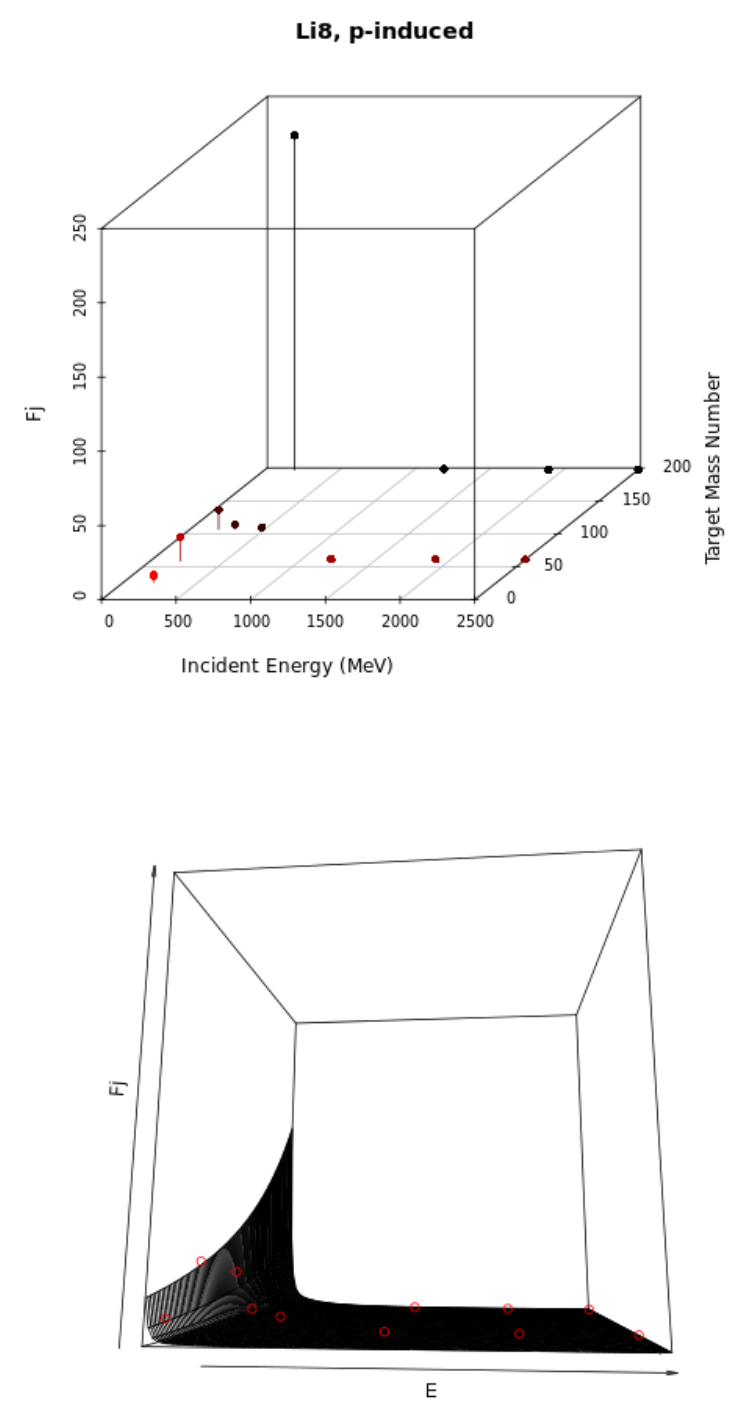

Figure 9: Values of $F_{j}$ according to incident proton energy $(\mathrm{MeV})$ and target mass number (top plot), shown with a corresponding surface mesh of the $F_{j}$ model, for ${ }^{8} \mathrm{Li}$.

therefore wish to obtain a $\gamma_{j}$ model that is both accurate and computationally fast. The $F_{j}$ model (and therefore, $\gamma_{j}$ model) accomplishes both of these: it is computationally simple and very fast, and it provides reasonably accurate fragment spectra compared to experimental results.

\section{Results}

In this section results comparing the $\gamma_{j}$ model with the new fitted $\gamma_{j}$ values are shown. In addition, results comparing the new $\gamma_{j}$ model within CEM03.03F with the old $\gamma_{j}$ model within CEM03.03 are shown.
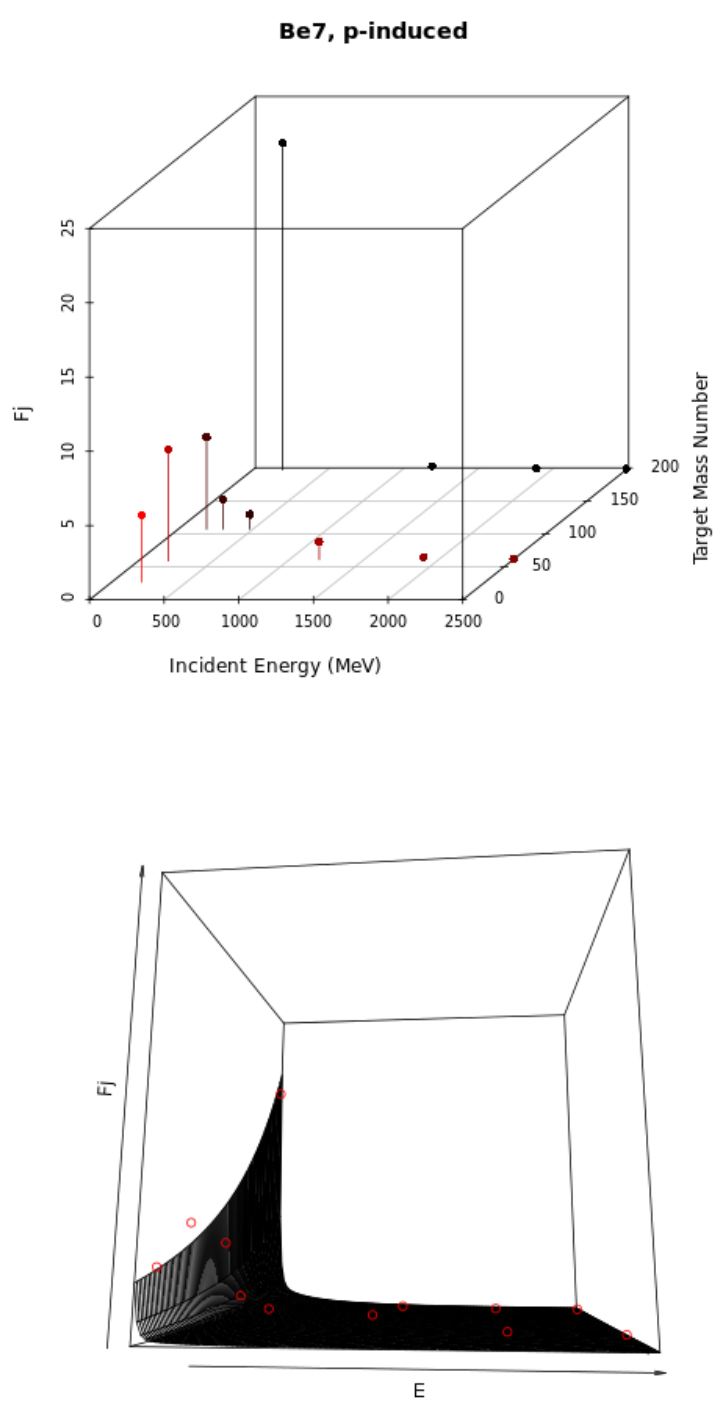

Figure 10: Values of $F_{j}$ according to incident proton energy (MeV) and target mass number (top plot), shown with a corresponding surface mesh of the $F_{j}$ model, for ${ }^{7} \mathrm{Be}$.

\subsection{Comparison of Spectra with Fitted $F_{j}$ vs. Spectra with $F_{j}$ Model}

For the large majority of reactions tested here, the predicted fragment spectra using the $F_{j}$ model was very similar to the predicted fragment spectra using fitted $F_{j}$ values. See Figs. 1315 for plots comparing results from the $F_{j}$ model with fitted $F_{j}$ values. Many more comparisons can be found in [20]. Oftentimes the $F_{j}$ model resulted in slightly "softer" spectra (i.e., ${ }^{6} \mathrm{Li}$ spectra from $200 \mathrm{MeV} \mathrm{p}+{ }^{197} \mathrm{Au}$, in Fig. 14), which was usually an improved match to experimental data than the $F_{j}$ fitted values.

There were a handful of reactions for which the predicted spectra of a few particular fragment types varied significantly: for the emission of heavier clusters $(A \geq 8)$ from reactions with low incident energies and heavy targets. For an example of this, 

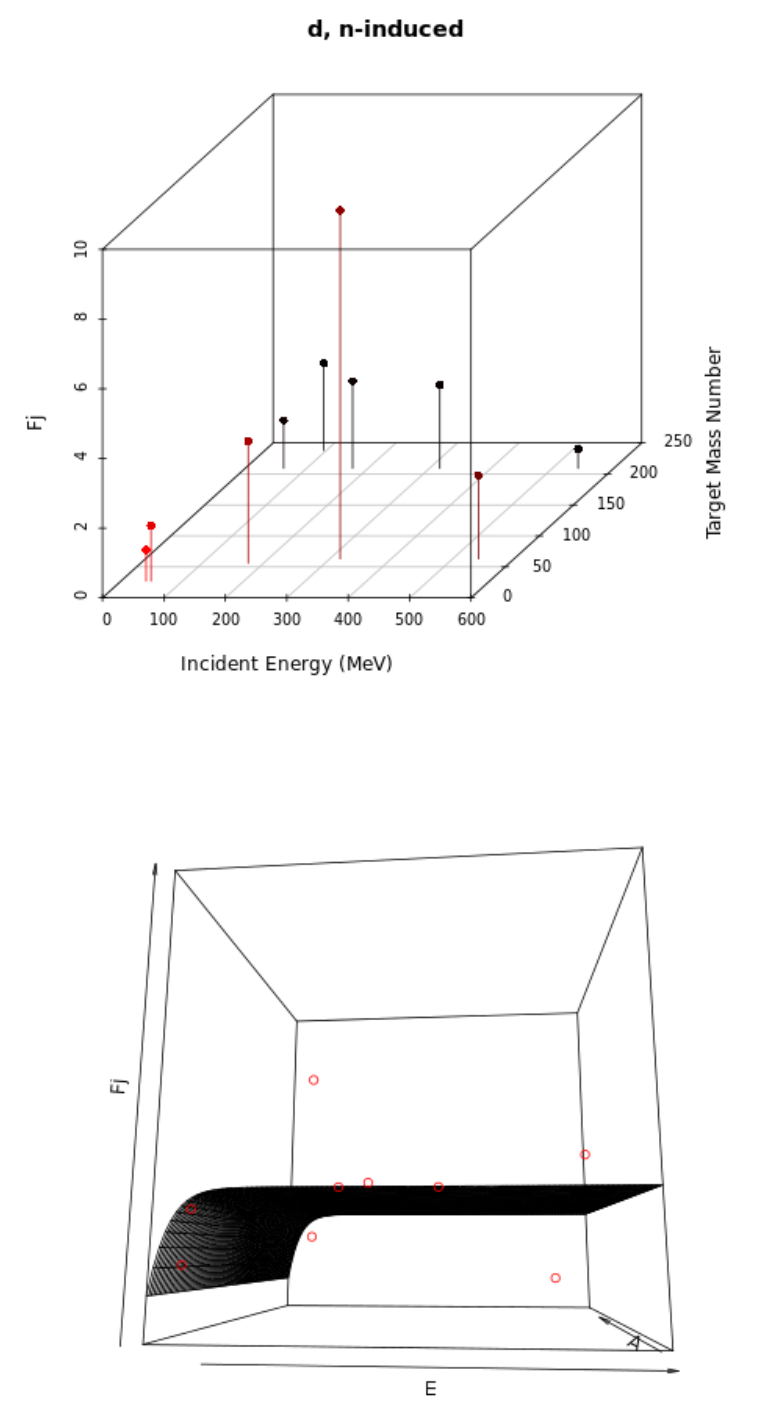

Figure 11: Values of $F_{j}$ according to incident neutron energy $(\mathrm{MeV})$ and target mass number (top plot), shown with a corresponding surface mesh of the $F_{j}$ model, for deuterium.

see the spectra of ${ }^{8} \mathrm{Li}$ in Fig. 14. While the discrepancy is significant, it is not always negative, as sometimes the fitted values were "overfit." We believe this difference is due to several factors. First, the expanded Coalescence model emits heavy clusters up to $A=7$; this leads to a "jump" in fitted $F_{j}$ values for emitted heavy clusters with $A>7$ as it is "compensating" for the lack of coalescence emission, compared to those with $A \leq 7$. However, as the $F_{j}$ model is a "smooth" model it cannot completely account for this jump. We expect that expanding the Coalescence model further will ease this effect. In addition, while we assumed for simplicity that the target-size dependence was independent of the fragment size and the incident energy, this is not strictly the case. The target-size dependence did, in fact, become more pronounced with increasing fragment size. We excluded target-size dependence from emitted ${ }^{4} \mathrm{He}$ and
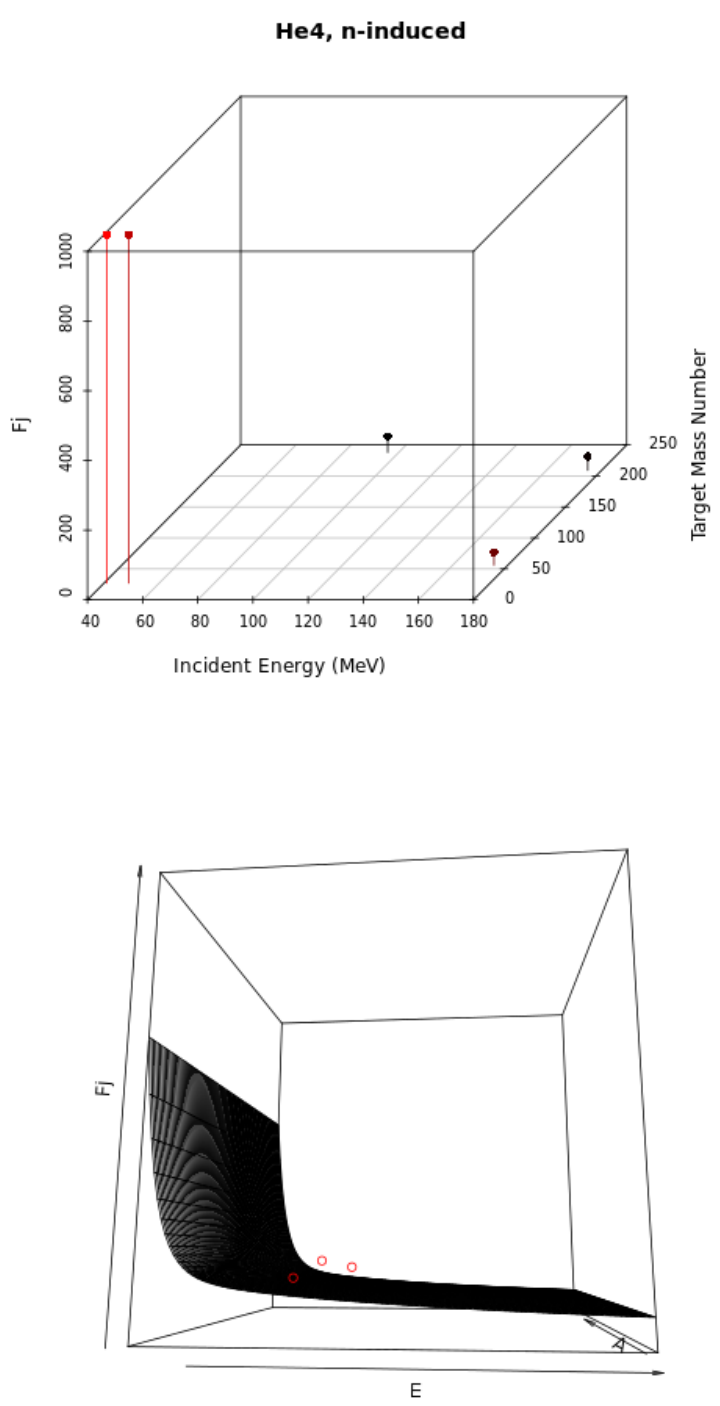

Figure 12: Values of $F_{j}$ according to incident neutron energy (MeV) and target mass number (top plot), shown with a corresponding surface mesh of the $F_{j}$ model, for ${ }^{4} \mathrm{He}$.

lighter, and included this term for heavier clusters, to partially account for this. However, the greater the fragment size the less valid this "constant" target-size dependence becomes. Lastly, we found that the target-size dependence also varied across the incident energy (also contrary to the assumption), becoming sharper at lower incident energies and flatter at higher incident energies. Thus, at low incident energies on heavy targets and looking at the emission of heavier clusters, we expect a "perfect storm" of factors to create significant discrepancy between the $F_{j}$ model and fitted values. However, as noted previously, this discrepancy is not always negative as sometimes it leads to either improved fit with experimental data or a more natural spectra. Furthermore, the ${ }^{8} \mathrm{Li}$ spectra of Fig. 14 demonstrate the need to also upgrade the evaporation model, as it primarily produces the peak of the spectra, which is too low for this reaction. 

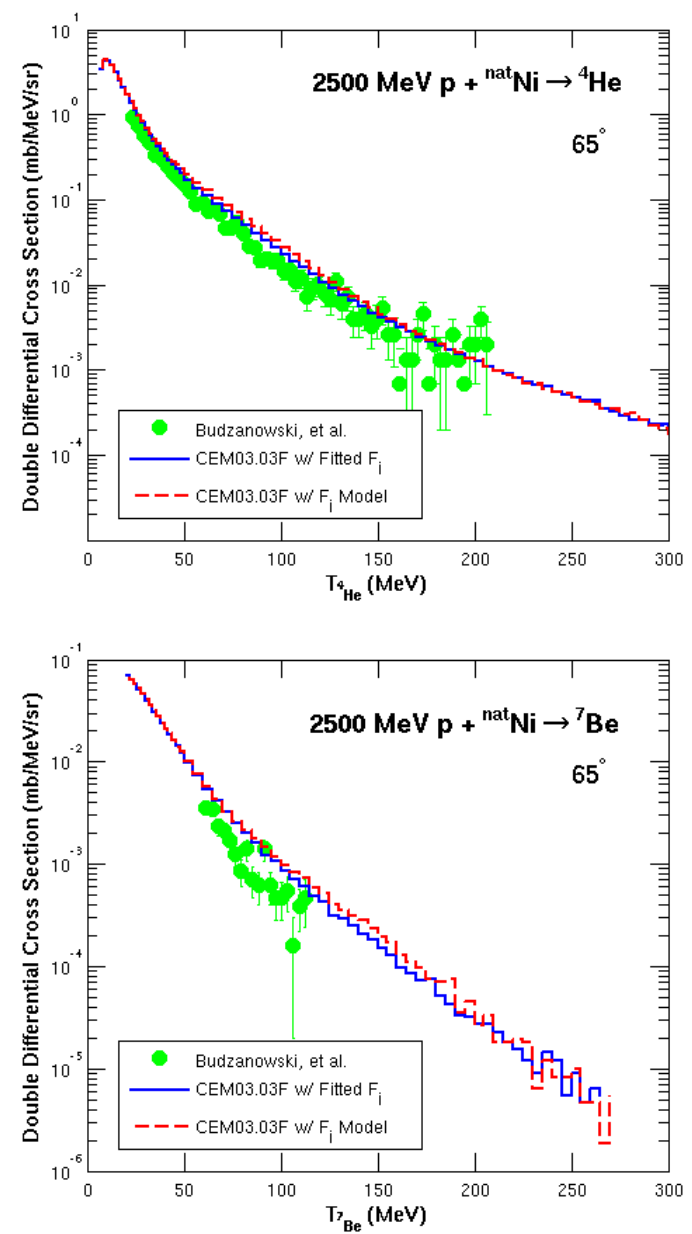

Figure 13: Comparison of experimental data by Budzanowski, et al. [24] (green circles) with results by the CEM03.03F with fitted $F_{j}$ (blue solid lines) and CEM03.03F with $F_{j}$ model (red dashed lines) for $2500 \mathrm{MeV} \mathrm{p}+{ }^{n a t} \mathrm{Ni} \rightarrow$ ${ }^{4} \mathrm{He},{ }^{7} \mathrm{Be}$.

We hope to do this in the future.

\subsection{Results of New CEM03.03F with $\gamma_{j}$ Model}

This section demonstrates the improved predictive capability of CEM03.03F with the new $\gamma_{j}$ model compared to CEM03.03 with the old $\gamma_{j}$ model. As displayed in Figs. 16-18, we observe significant improvement in the production of heavy clusters $\left(>^{4} \mathrm{He}\right)$, owing in part to the fact that the old model was not designed for fragments heavier than ${ }^{4} \mathrm{He}$. The difference is also due to several other heavy-ion upgrades included in CEM03.03F $[8,9,11]$; however, these other improvements would be crippled by the old $\gamma_{j}$ model.

Fig. 16 illustrates our results for $200 \mathrm{MeV} p+{ }^{59} \mathrm{Co} \rightarrow{ }^{9} \mathrm{Li}$ at $60^{\circ}$ with experimental data by Machner, et al. [25]. This figure demonstrates results for the rare, neutron-rich lithium isotope ${ }^{9} \mathrm{Li}$. We see dramatic improvement in the production of highenergy ${ }^{9} \mathrm{Li}$ with CEM03.03F.

We also created a working version of MCNP6 (that we call MCNP6-F) which contains our CEM03.03F heavy-ions upgrades, including the new $\gamma_{j}$ model discussed in this paper.
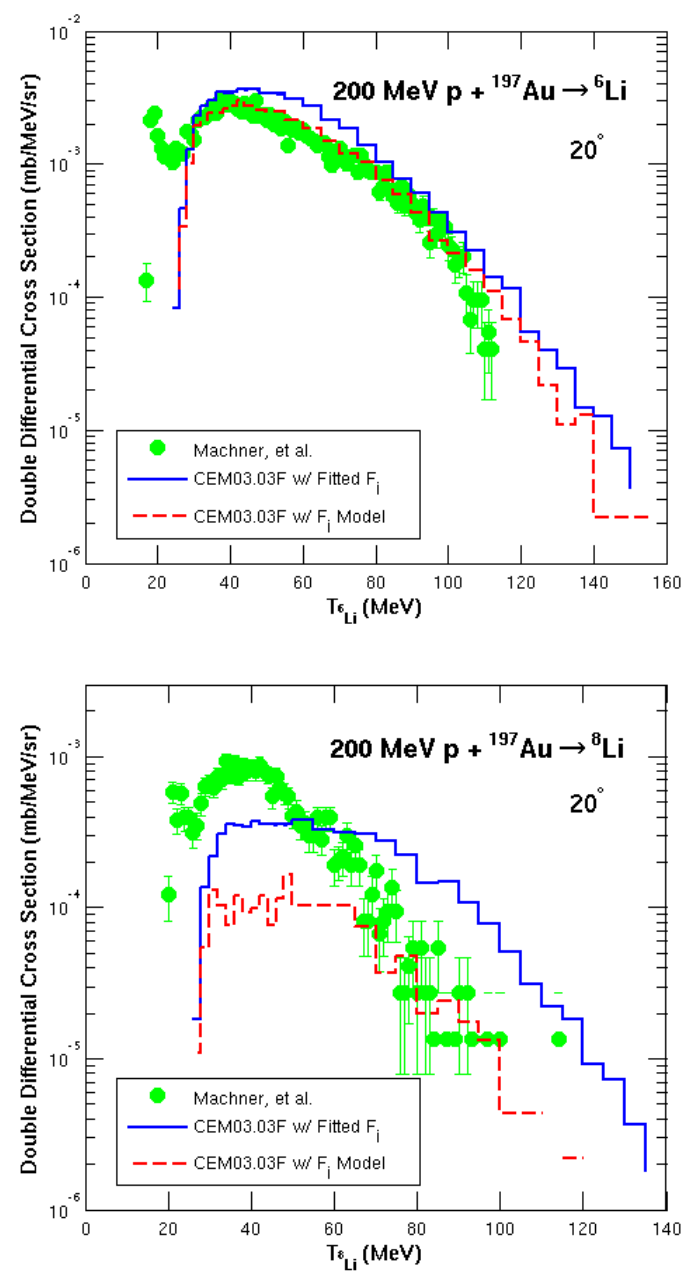

Figure 14: Comparison of experimental data by Machner, et al. [25] (green circles) with results by the CEM03.03F with fitted $F_{j}$ (blue solid lines) and CEM03.03F with $F_{j}$ model (red dashed lines) for $200 \mathrm{MeV} \mathrm{p}+{ }^{197} \mathrm{Au} \rightarrow{ }^{6} \mathrm{Li}$, ${ }^{8} \mathrm{Li}$

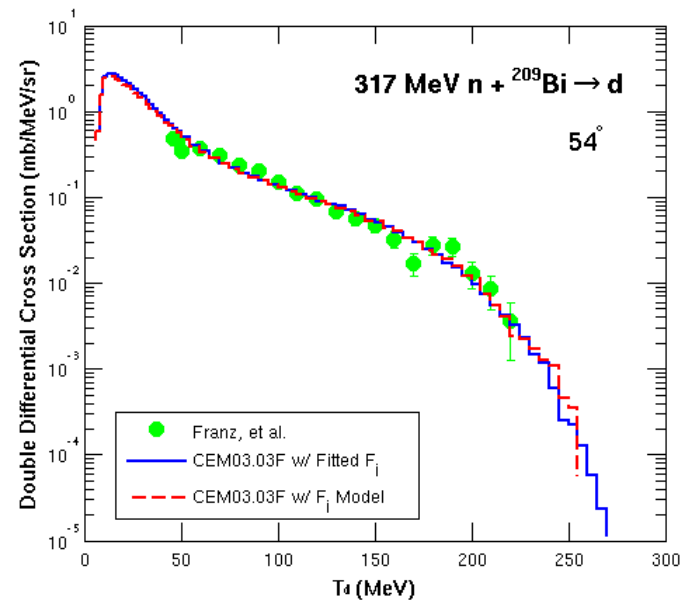

Figure 15: Comparison of experimental data by Franz, et al. [? ] (green circles) with results by the CEM03.03F with fitted $F_{j}$ (blue solid lines) and CEM03.03F with $F_{j}$ model (red dashed lines) for $317 \mathrm{MeV} \mathrm{n}+{ }^{209} \mathrm{Bi} \rightarrow \mathrm{d}$ 


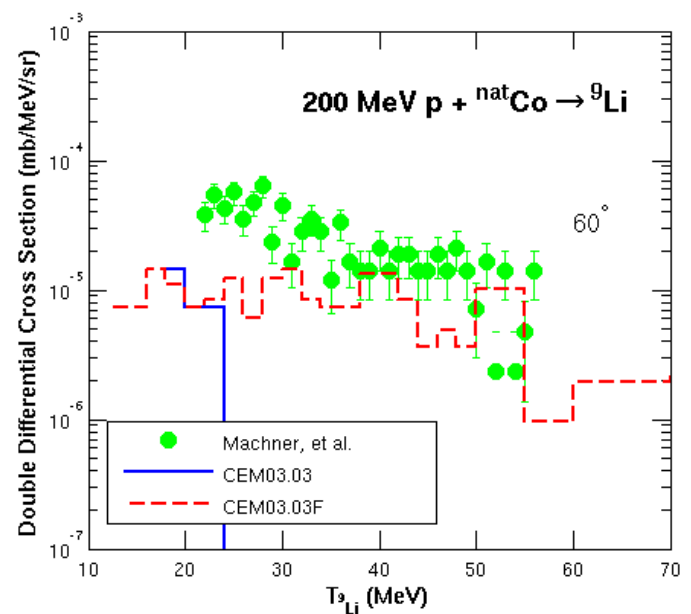

Figure 16: Comparison of experimental data by Machner, et al. [25] (green circles) with results by CEM03.03 with the old $\gamma_{j}$ model (blue solid lines) and new CEM03.03F with the new $\gamma_{j}$ model (red dashed lines) for $200 \mathrm{MeV} \mathrm{p}+$ ${ }^{9} \mathrm{Co} \rightarrow{ }^{9} \mathrm{Li}$ at $60^{\circ}$

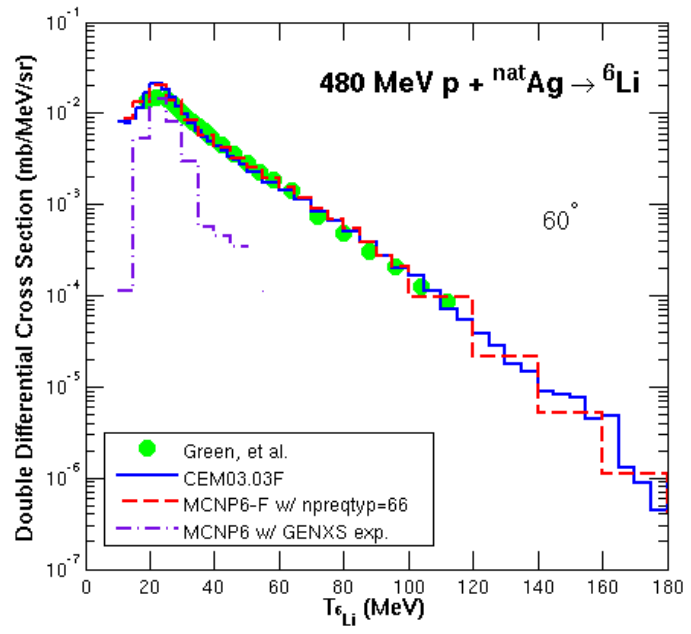

Figure 17: Comparison of experimental data by Green, et al. [26] (green circles) with results by CEM03.03F (blue solid lines), MCNP6-F with npreqtyp=66 (red dashed lines), and MCNP6 with the GENXS expansion only (purple dash-dotted lines) for $480 \mathrm{MeV} \mathrm{p}+{ }^{n a t} \mathrm{Ag} \rightarrow{ }^{6} \mathrm{Li}$ at $60^{\circ}$.

Fig. 17 shows our results for $480 \mathrm{MeV} \mathrm{p}+{ }^{n a t} \mathrm{Ag} \rightarrow{ }^{6} \mathrm{Li}$ at $60^{\circ}$ with experimental data by Green, et al. [26]. We see that MCNP6-F produces significantly improved results and matches the data reasonably well.

Fig. 18 displays our results for $1200 \mathrm{MeV} p+{ }^{197} \mathrm{Au} \rightarrow{ }^{6} \mathrm{Li}$ at $20^{\circ}$ with experimental data by Budzanowski, et al. [27]. Again CEM03.03F demonstrates dramatically increased production of heavy clusters in the mid- and high-energy regions compared to the original CEM03.03.

We also see improvement in the production of fragments up to ${ }^{4} \mathrm{He}$, which the old $\gamma_{j}$ model included. Fig. 19 displays our results for $660 \mathrm{MeV} \mathrm{p}+{ }^{58} \mathrm{Ni} \rightarrow{ }^{4} \mathrm{He}$ at $90^{\circ}$ with experimental data by Bogatin, et al. [28]. This figure demonstrates that CEM03.03F achieves improved prediction of spectra even for

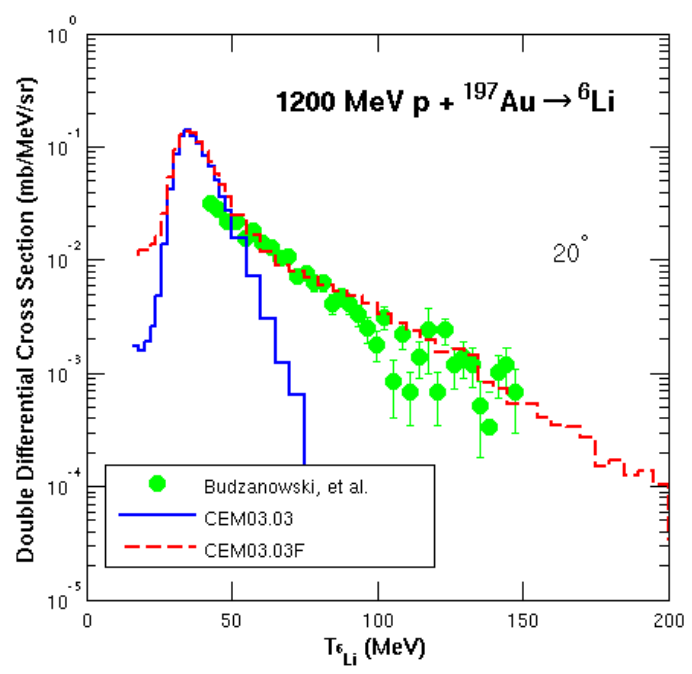

Figure 18: Comparison of experimental data by Budzanowski, et al. [27] (green circles) with results by CEM03.03 with the old $\gamma_{j}$ model (blue solid lines) and new CEM03.03F with the new gamma ${ }_{j}$ model (red dashed lines) for $1200 \mathrm{MeV}$ $\mathrm{p}+{ }^{197} \mathrm{Au} \rightarrow{ }^{6} \mathrm{Li}$ at $20^{\circ}$.

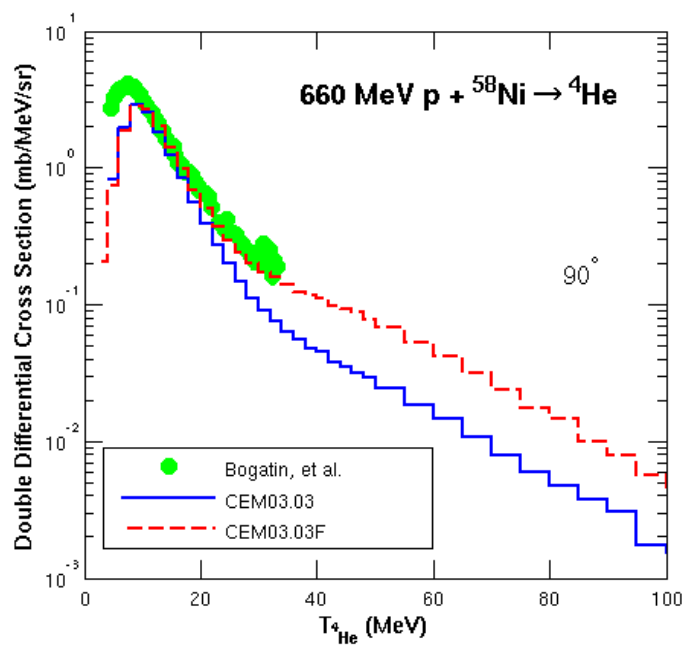

Figure 19: Comparison of experimental data by Bogatin, et al. [28] (green circles) with results by CEM03.03 with the old $\gamma_{j}$ model (blue solid lines) and new CEM03.03F with the new gamma ${ }_{j}$ model (red dashed lines) for $660 \mathrm{MeV}$ $\mathrm{p}+{ }^{58} \mathrm{Ni} \rightarrow{ }^{4} \mathrm{He}$ at $90^{\circ}$.

fragments $\leq{ }^{4} \mathrm{He}$, which the old $\gamma_{j}$ model produces reasonably well.

\section{Conclusion}

While $\gamma_{j}$ can theoretically be calculated from first principles, this is too computationally time-consuming. We therefore wish to obtain a $\gamma_{j}$ model that is both accurate and computationally fast. The $F_{j}$ model (and therefore, $\gamma_{j}$ model) accomplishes both of these: it is computationally simple and very fast, and it provides reasonably accurate fragment spectra compared to experimental results. 
The $\gamma_{j}$ model is dependent upon incident projectile energy, target nucleus size, and emitted fragment size. We found two different energy dependencies: one for the low-energy region and a second for the higher-energy region. These energy dependencies make sense considering the physics of nuclear reactions. We consider in this $F_{j}$ model the incident energy of the incoming proton, not the excitation energy of the residual nucleus at the time of preequilibrium, and after a certain incident energy we expect the residual nucleus energy to not change significantly as the incident energy continues to increase: i.e., the amount of energy deposited in the target nucleus reaches a saturating limit and increasing the incident proton energy further does not lead to significantly greater residual nucleus energy. This idea is analogous to "limiting fragmentation" $[22,23]$. In addition, CEM accounts for the IntraNuclear Cascade, preequilibrium, evaporation/fission, Fermi break-up, and coalescence mechanisms of nuclear reactions, but does not account for pickup and knock-out reactions. Pick-up and knock-out mechanisms are especially important at low energies, and therefore, the increase in $F_{j}$ at lower incident energies can be attributed to "compensating" for these missing physics in CEM. Furthermore, CEM does not account for some nuclear structure effects important at low energies, and for some reactions, also at high energies. Lastly, CEM, just as any other model, is "only a model," and probably misses some other aspects of the physics. For these reasons, we need to look at the "model" for $\gamma_{j}$ in CEM as heuristic, understanding that in some energy/target-size regions, it does not have exactly the right meaning of the condensation probability, but also contains a component to counterbalance physics not accounted for in CEM.

This $\gamma_{j}$ model is specifically designed for use in CEM and MCNP6, taking into account the reaction mechanisms used (or not used) in CEM. However, this model could be useful in other nuclear spallation codes and models, especially for heavy cluster production.

In conclusion, the new $\gamma_{j}$ model provides better agreement with experimental data than the old interpolation fits used in CEM03.03, especially for heavy-cluster spectra. We plan to apply this to LAQGSM as well, and implement the new model within MCNP6.

\section{Acknowledgments}

I am grateful to my colleague, Dr. Stepan G. Mashnik, for a long and very fruitful collaboration with me and for several useful discussions of the results presented here.

I am grateful to Drs. Avneet Sood, Chris Werner, and Lawrence Cox of Los Alamos National Laboratory and to Prof. Akira Tokuhiro of the University of Idaho for encouraging discussions and support.

This study was carried out under the auspices of the National Nuclear Security Administration of the U.S. Department of Energy at Los Alamos National Laboratory under Contract No. DE-AC52-06NA25396.

This work is supported in part by the M. Hildred Blewett
Fellowship of the American Physical Society, www.aps.org.

\section{References}

[1] T. Goorley, M. James, T. Booth, F. Brown, J. Bull, L. J. Cox, J. Durkee, J. Elson, M. Fensin, R. A. Forster, J. Hendricks, H. G. Hughes, R. Johns, B. Kiedrowski, R. Martz, S. Mashnik, G. McKinney, D. Pelowitz, R. Prael, J. Sweezy, L. Waters, T. Wilcox, and T. Zukaitis, Initial MCNP6 Release Overview, MCNP6 version 0.1, Nuclear Technology 180 (2012) 298.

[2] K. K. Gudima, S. G. Mashnik, and V. D. Toneev, Cascade-Exciton Model of Nuclear Reactions, Nuclear Physics A401 (1983) 329.

[3] S. G. Mashnik, K. K. Gudima, R. E. Prael, A. J. Sierk, M. I. Baznat, and N. V. Mokhov, CEM03.03 and LAQGSM03.03 Event Generators for the MCNP6, MCNPX, and MARS15 Transport Codes, Joint ICTP-IAEA Advanced Workshop on Model Codes for Spallation Reactions. Trieste, Italy, February 2008, LANL Report, LA-UR-08-2931. arXiv:0805.0751.

[4] K. K. Gudima, S. G. Mashnik, and A. J. Sierk, User Manual for the Code LAQGSM, LANL Report LA-UR-01-6804; http://libwww.lanl.gov/lapubs/00818645.pdf.

[5] E. Betak, Complex Particle Emission in the Exciton Model of Nuclear Reactions, Acta Physica Slovaka 26 (1976) 21.

[6] C. Cline, Extensions to the Pre-Equilibrium Statistical Model and a Study of Complex Particle Emission, Nuclear Physics A 193 (1972) 417.

[7] I. Ribansky, P. Oblozinsky, and E. Betak, Pre-Equilibrium Decay and the Exciton Model, Nucl. Phys. A205 (1973) 545.

[8] L. M. Kerby and S. G. Mashnik, Production of Heavy Clusters with an Expanded Coalescence Model in CEM, Transactions 112 (2015) 577.

[9] L. M. Kerby and S. G. Mashnik, Total Reaction Cross Sections in CEM and MCNP6 at Intermediate Energies, Nuclear Instruments and Methods B 356-357 (2015) 135; LANL Report, LA-UR-14-29371; arXiv: 1505.00842 .

[10] S. Mashnik, L. Kerby, MCNP6 Fragmentation of Light Nuclei at Intermediate Energies, Nuclear Instruments and Methods in Physics Research A 764 (2014) 59; arXiv:1404.7820.

[11] L. M. Kerby, S. G. Mashnik, and A. T. Tokuhiro, L. Kerby, S. Mashnik, A. Tokuhiro, Production of Energetic Light Fragments with Expanded Cascade Exciton Model (CEM), Transactions of the American Nuclear Society 110 (2014) 465.

[12] L. M. Kerby, S. G. Mashnik, A. J. Sierk, Preequilibrium Emission of Light Fragments in Spallation Reactions, Nuclear Data Sheets 118 (2014) 316; arXiv:1303.4311.

[13] K. K. Gudima, G. A. Ososkov, and V. D. Toneev, Model for PreEquilibrium Decay of Excited Nuclei, Yadernaya Fizika 21 (1975) 260, [Soviet Journal of Nuclear Physics 21 (1975) 139].

[14] S. G. Mashnik and V. D. Toneev, MODEX-the Program for Calculation of the Energy Spectra of Particles Emitted in the Reactions of PreEquilibrium and Equilibrium Statstical Decays, JINR Communication P4-8417 (1974)

[15] S. G. Mashnik, K. K. Gudima, A. J. Sierk, M. I. Baznat, N. V. Mokhov, CEM03.01 User Manual, LANL Report LA-UR-05-7321, Los Alamos 2005; https://mcnp.lanl.gov/.

[16] I. Ribansky and P. Oblozinsky, Emission of Complex Particles in the Exciton Model, Physics Letters 45B(4) (1973).

[17] J. R. Wu and C. C. Chang, Complex-Particle Emission in the PreEquilibrium Exciton Model, Physical Review C 17 (1978) 1540.

[18] V. Blideanu, F. R. Lecolley, J. F. Lecolley, T. Lefort, N. Marie, A. Ataç, G. Ban, B. Bergenwall, J. Blomgren, S. Dangtip, K. Elmgren, Ph. Eudes, Y. Foucher, A. Guertin, F. Haddad, A. Hildebrand, C. Johansson, O. Jonsson, M. Kerveno, T. Kirchner, J. Klug, Ch. Le Brun, C. Lebrun, M. Louvel, P. Nadel-Turonski, L. Nilsson, N. Olsson, S. Pomp, A. V. Prokofiev, P.-U. Renberg, G. Rivière, I. Slypen, L. Stuttgé, U. Tippawan, and M. Österlund, Nucleon-induced reactions at intermediate energies: New data at $96 \mathrm{MeV}$ and theoretical status, Physical Review C 70 (2004) 014607.

[19] L. M. Kerby, Precompound Emission of Energetic Light Fragments in Spallation Reactions, Ph. D. Thesis, University of Idaho, August 2015; LANL Report LA-UR-15-23856.

[20] L.M. Kerby and S.G. Mashnik, A New Model for the Condensation Probability, $\gamma_{j}$, in CEM, LANL Report, LA-UR-15-22370, April 2015.

[21] W. N. Venables, D. M. Smith, and the R Core Team, An Introduction to R. 
Notes on R: A Programming Environment for Data Analysis and Graphics Version 3.2.0 (2015-04-16), http://cran.r-project.org/doc/manuals/rrelease/R-intro.pdf.

[22] J. Benecke, T. T. Chou, C. N. Yang, and E. Yen, Hypothesis of Limiting Fragmentation in High-Energy Collisions, Physical Review 188 (1969) 2159.

[23] M.S. El-Nagdy, A. Abdelsalam, Z. Abou-Moussa, and B.M. Badawy, Limiting fragmentation of target nucleus at high energy, Canadian Journal of Physics, 91 (2013) 737-743.

[24] A. Budzanowski, M. Fidelus, D. Filges, F. Goldenbaum, H. Hodde, L. Jarczyk, B. Kamys, M. Kistryn, S. Kistryn, S. Kliczewski, A. Kowalczyk, E. Kozik, P. Kulessa, H. Machner, A. Magiera, B. Piskor-Ignatowicz, K. Pysz, Z. Rudy, R. Siudak, and M. Wojciechowski, Comparison of Nonequilibrium Processes in $\mathrm{p}+\mathrm{Ni}$ and $\mathrm{p}+\mathrm{Au}$ Collisions at $\mathrm{GeV}$ Energies, Physical Review C 82 (2010) 034605.

[25] H. Machner, D. G. Aschman, K. Baruth-Ram, J. Carter, A. A. Cowley, F. Goldenbaum, B. M. Nangu, J. V. Pilcher, E. Sideras-Haddad, J. P. F. Sellschop, F. D. Smit, B. Spoelstra, and D. Steyn, Isotopic Production Cross Sections in Proton-Nucleus Collisions at $200 \mathrm{MeV}$, Physical Review C 73 (2006) 044606.

[26] R. E. L. Green, R. G. Korteling, and K. P. Jackson, Inclusive Production of Isotopically Resolved Li Through Mg Fragments by $480 \mathrm{MeV}$ p+Ag Reactions, Physical Review C 29 (1984) 1806.

[27] A. Budzanowski, M. Fidelus, D. Filges, F. Goldenbaum, H. Hodde, L. Jarczyk, B. Kamys, M. Kistryn, S. Kistryn, S. Kliczewski, A. Kowalczyk, E. Kozik, P. Kulessa, H. Machner, A. Magiera, B. Piskor-Ignatowicz, K. Pysz, Z. Rudy, R. Siudak, and M. Wojciechowski, Competition of Coalescence and "Fireball" Processes in Nonequilibrium Emission of Light Charged Particles from p+Au Collisions, Phys. Rev. C 78 (2008) 024603.

[28] V. I. Bogatin, V. F. Litvin, O. V. Lozhkin, N. A. Perfilov, and Yu. P. Yakovlev, Isotopic Effects in High-Energy Nuclear Reactions and Isospin Correlations of Fragmentation Cross Sections, Nuclear Physics A 260 (1976) 446. 